\title{
Long-Range Coherence and Multiple Steady States in a Lossy Qubit Array
}

\author{
Shovan Dutta $\odot^{*}$ and Nigel R. Cooper ${ }^{\dagger}$ \\ T. C. M. Group, Cavendish Laboratory, University of Cambridge, JJ Thomson Avenue, Cambridge CB3 OHE, United Kingdom
}

(Received 16 April 2020; accepted 11 November 2020; published 9 December 2020)

\begin{abstract}
We show that a simple experimental setting of a locally pumped and lossy array of two-level quantum systems can stabilize states with strong long-range coherence. Indeed, by explicit analytic construction, we show there is an extensive set of steady-state density operators, from minimally to maximally entangled, despite this being an interacting open many-body problem. Such nonequilibrium steady states arise from a hidden symmetry that stabilizes Bell pairs over arbitrarily long distances, with unique experimental signatures. We demonstrate a protocol by which one can selectively prepare these states using dissipation. Our findings are accessible in present-day experiments.
\end{abstract}

DOI: 10.1103/PhysRevLett.125.240404

Introduction.-Coupling a quantum system to an environment typically results in a loss of coherence [1], which is a major obstacle for quantum control and information processing [2-4]. However, a growing number of studies have shown that a well-designed coupling can also drive the system toward interesting and useful quantum states [5-7]. This is particularly promising in light of parallel advances in experimental platforms where one can engineer both the system Hamiltonian and the coupling [8-12], offering novel out-of-equilibrium settings where interactions and dissipation compete $[13,14]$.

A rare phenomenon occurs when such an open quantum system has multiple stable states owing to a symmetry that gives a conserved quantum number $[15,16]$. Then the dynamics decouple into independent sectors [17], with the system retaining some memory of its initial state [18]. Furthermore, one can show that information encoded in the steady-state manifold would be preserved unconditionally [19], and one could control transport by switching between the symmetry sectors [20]. So far, this kind of strong symmetry has been found only theoretically in symmetric networks [21-23] and in boundary-driven spin chains with nonstandard dissipation [17,24], without experimental realizations. They require special design even in noninteracting systems [23].

In this Letter, we identify a prototypical setting of a simple lattice model with a routine bulk dissipation that possesses a surprising hidden symmetry, leading to multiple steady states with long-range coherence and nonlocal Bell pairs. The steady states can be selectively prepared and probed in existing setups. To illustrate our findings, we model hard-core bosons on a one-dimensional (1D) lattice, which is equivalent to an array of qubits or an (anisotropic) spin chain. Our conclusions extend more generally to a broad class of models of this type.

We consider hard-core bosons on a lattice with particle injection and loss at two sites. Such a local incoherent pump was used recently to prepare a Mott insulator of photons [25]. When the source and sink are at the boundary, the system can be reduced to free fermions [26-28]. However, for dissipation in the bulk, such a reduction is not possible, and the system is strongly interacting. We focus on the special case where the pump and loss both act on the center site. Beyond the obvious reflection parity, we find a dynamical symmetry that can be roughly interpreted as conserving a total "charge" of symmetrically located particle-hole Bell pairs. Consequently, the number of symmetry sectors grows linearly with the system size $L$, yielding an extensive degeneracy. We provide an exact solution for the steady-state manifold and show that it includes a maximally entangled sector with $(L-1) / 2$ nonlocal Bell pairs. We demonstrate a procedure for preparing the system in any given sector. Subsequent dynamics within the sector converge to a unique steady state, which can be discerned by measuring single-particle or density correlations [29-31]. Additionally, in the limit of zero pump (or loss) rate, the degeneracy is increased further to accommodate a decoherence-free subspace, a key ingredient for quantum computing [32].

Model.-We study hard-core bosons [33] hopping on a 1D lattice with an odd number of sites, $L:=2 l+1$ for integer $l$, described by the Hamiltonian

$$
\hat{H}=-\hbar J \sum_{i=-l}^{l-1}\left(\hat{b}_{i}^{\dagger} \hat{b}_{i+1}+\hat{b}_{i+1}^{\dagger} \hat{b}_{i}\right)
$$

where $J$ is the hopping amplitude and $\hat{b}_{i}^{\dagger}$ creates a boson at site $i$. The hard-core condition is imposed by requiring $\hat{b}_{i}^{\dagger 2}=0$, which ensures there can be either 0 or 1 particle at any given site. This regime corresponds to the stronginteraction limit of the Bose-Hubbard model [34] and has been realized with atoms in optical lattices [35-37] and with photons in nonlinear resonators [25]. The hard-core 
constraint implies the commutation rules $\left[\hat{b}_{i}, \hat{b}_{j}\right]=0$ and $\left[\hat{b}_{i}, \hat{b}_{j}^{\dagger}\right]=(-1)^{\hat{n}_{i}} \delta_{i j}$, where $\hat{n}_{i}:=\hat{b}_{i}^{\dagger} \hat{b}_{i}$ is the occupation at site $i$ [38]. The Hamiltonian maps onto free fermions by a Jordan-Wigner (JW) transformation [39]:

$$
\hat{f}_{j}=(-1)^{\sum_{i<j} \hat{n}_{i}} \hat{b}_{j} ; \quad \hat{b}_{j}=(-1)^{\sum_{i<j} \hat{n}_{i}} \hat{f}_{j},
$$

where $\hat{f}_{j}$ are fermionic operators that satisfy anticommutation, $\left\{\hat{f}_{i}, \hat{f}_{j}\right\}=0$ and $\left\{\hat{f}_{i}, \hat{f}_{j}^{\dagger}\right\}=\delta_{i j}$. Thus, $\hat{f}_{i}^{\dagger} \hat{f}_{i}=\hat{n}_{i}$, and Eq. (1) is restated as $\hat{H}=-\hbar J \sum_{i}\left(\hat{f}_{i}^{\dagger} \hat{f}_{i+1}+\right.$ H.c. $)$. However, as we show below, the dissipation mediates interactions between these fermion operators.

We add dissipation by coupling the system of bosons to bosonic reservoirs that inject particles at site $p$ and remove particles from site $q$. The reservoirs have a finite bandwidth, such that if site $p$ is already occupied, further injection is suppressed by the large interaction energy. Such local sources and sinks have been engineered using transmon qubits in microwave circuits [25]. Typically, in these photonic setups, the reservoirs relax to equilibrium much faster than the system dynamics [40]. Under such a routine Born-Markov approximation, the reduced density matrix $\hat{\rho}$ of the system is governed by a master equation of the Lindblad form [34,40-46]

$\frac{d \hat{\rho}}{d t}=\mathcal{L} \hat{\rho}:=-\frac{i}{\hbar}[\hat{H}, \hat{\rho}]+\sum_{\alpha} \hat{L}_{\alpha} \hat{\rho} \hat{L}_{\alpha}^{\dagger}-\frac{1}{2}\left\{\hat{L}_{\alpha}^{\dagger} \hat{L}_{\alpha}, \hat{\rho}\right\}$,

where $\hat{L}_{1}:=\sqrt{\gamma_{+}} \hat{b}_{p}^{\dagger}$ and $\hat{L}_{2}:=\sqrt{\gamma_{-}} \hat{b}_{q}$ are two Lindblad operators, $\gamma_{ \pm}$being the pump and loss rates, respectively. This dynamics could also be realized with cold atoms by mapping the system to a spin- $1 / 2 X X$ chain with local incoherent spin flips [33]. Such a chain could be engineered with either motional states [47] or internal states [48-50], using local addressability to flip spins [51]. Note our main results do not depend on the exact form of the Lindblad operators as long as they are local.

If the pump and loss are at the ends of the chain (i.e., $|p|=|q|=l$ ), the problem reduces to a description in which the Liouvillian $\mathcal{L}$ is quadratic in the JW fermions and the system is noninteracting [26-28,52]. If either pump or loss occurs in the bulk, this can no longer be achieved. Then $\mathcal{L}$ contains terms involving string operators $(-1)^{\hat{N}_{L}}$, where $\hat{N}_{L}$ is the number of particles to the left of the dissipation site, which is not conserved by the Hamiltonian. Consequently, $\mathcal{L}$ is not quadratic, and the system is genuinely interacting.

Here, we focus on these cases where pump or loss does not occur at the boundary. For such interacting systems, one expects that, under generic conditions, Eq. (3) has a unique steady state [55-57]. We find this is indeed the case if the pump or loss occurs at any site other than the center [59]. For $p=q \neq 0$, the system reaches a product state $\hat{\rho}=\otimes_{i}\left(\gamma_{+}\left|1_{i}\right\rangle\left\langle 1_{i}\left|+\gamma_{-}\right| 0_{i}\right\rangle\left\langle 0_{i}\right|\right) /\left(\gamma_{+}+\gamma_{-}\right)$[60]. The situation is very different, however, if the pump and loss are both at the center site, unlocking multiple "strong" symmetries [17] and leading to many striking effects.

Hidden symmetry.-To understand the symmetries that arise when both pump and loss occur at the center, $p=$ $q=0$, consider first the reflection symmetry. Reflections are generated by an operator $\hat{R}$ that exchanges sites $i$ and $-i$ for all $i$, such that $\hat{R} \hat{b}_{i} \hat{R}=\hat{b}_{-i}$. One can readily show that $\hat{R}$ commutes with both the Hamiltonian and the dissipators:

$$
[\hat{H}, \hat{R}]=0 \quad \text { and } \quad\left[\hat{L}_{\alpha}, \hat{R}\right]=0 \quad \forall \alpha,
$$

the latter arising since the dissipators involve only $\hat{b}_{0}^{(\dagger)}$ and $\left[\hat{b}_{0}^{(\dagger)}, \hat{R}\right]=0$. Consequently, reflection $\hat{R}$ generates a socalled "strong" symmetry [17] and leads to multiple steady states. Here, the system evolves separately in its even- and odd-parity sectors, giving rise to (at least) two steady states associated with the two parities.

The dynamics are far more constrained, however, by a hidden symmetry [61] generated by another operator $\hat{C}^{2}$, where

$$
\hat{C}:=-1 / 2+\sum_{k=-l}^{l} \hat{f}_{k}^{\dagger} \hat{f}_{-k} .
$$

From Eq. (2), every $k \neq 0$ term in $\hat{C}$ contains the factor $(-1)^{\hat{n}_{0}}$, and the remaining terms give $\hat{n}_{0}-1 / 2 \propto(-1)^{\hat{n}_{0}}$. Thus, $\left[\hat{b}_{0}^{(\dagger)}, \hat{C}^{2}\right]=0$; i.e., $\hat{C}^{2}$ commutes with the dissipators $\hat{L}_{1,2}$. Furthermore, as we show in the Supplemental Material [62], $\hat{C}=\hat{N}_{\text {even }}-\hat{N}_{\text {odd }}-1 / 2$, where $\hat{N}_{\text {even }}$ and $\hat{N}_{\text {odd }}$ are the total occupations of the even and odd singleparticle energy modes, respectively, which gives $[\hat{H}, \hat{C}]=$ 0 [65]. Therefore, $\hat{C}^{2}$ generates a strong symmetry. Note this is an exact result for the hard-core bosons. One also finds $\hat{C}$ is symmetric under reflection about the center, and all of its eigenspaces have a definite $\hat{R}$ parity.

In general, the eigenspaces of a strong symmetry generator $\hat{S}$ evolve independently, each having at least one steady state [17]. This decoupling originates from conservation laws. In particular, using $\langle\hat{S}\rangle=\operatorname{Tr}(\hat{\rho} \hat{S})$ in Eq. (3), one finds $d\langle\hat{S}\rangle / d t=i\langle[\hat{H}, \hat{S}]\rangle / \hbar-\sum_{\alpha} \operatorname{Re}\left\langle\hat{L}_{\alpha}^{\dagger}\left[\hat{L}_{\alpha}, \hat{S}\right]\right\rangle=0$; i.e., $\langle\hat{S}\rangle$ is conserved $[16,66]$. Moreover, the projectors onto each of the eigenspaces of $\hat{S}$ satisfy Eq. (4) individually and are conserved separately [15]. In other words, the weight in each symmetry sector is preserved.

Here, there will appear multiple steady states associated with the different eigenspaces of $\hat{C}^{2}$. As we explain below, the eigenstates of $\hat{C}^{2}$ comprise entangled particle-hole pairs at sites $k$ and $-k$, each carrying a quantum number taking values \pm 1 that we call "charge." The full spectrum consists of $l+1$ eigenvalues, $\left\{(\eta+1 / 2)^{2}: \eta=0, \ldots, l\right\}$, where $\eta$ is 
a measure of the total charge of all such pairs. These eigenspaces evolve independently, and we find every sector has a unique steady state for $\gamma_{ \pm} \neq 0$, leading to an $(l+1)$ fold degeneracy. This is in sharp contrast to the noninteracting problem, where free bosons [67] or fermions are subject to pump or loss at the center. Then, every odd single-particle state is unaffected by dissipation so its occupation number is conserved, yielding an exponentially large decoherence-free subspace of degenerate steady states. Later, we will use this feature for preparing the symmetry sectors of $\hat{C}^{2}$.

Steady states.-We first characterize the eigenspaces of $\hat{C}$ which is written as a sum of $l+1$ commuting parts, $\hat{C}_{0}:=\hat{n}_{0}-1 / 2$ and $\hat{C}_{k}:=\hat{f}_{k}^{\dagger} \hat{f}_{-k}+$ H.c. for $k=1, \ldots, l$. The latter describes hopping of JW fermions between two sites and can be diagonalized as $\hat{C}_{k}=\sum_{s= \pm} s \hat{a}_{k, s}^{\dagger} \hat{a}_{k, s}$, where $\hat{a}_{k, \pm}:=\left(\hat{f}_{k} \pm \hat{f}_{-k}\right) / \sqrt{2}$ are single-particle fermion modes. Thus, $\hat{C}_{k}$ has eigenstates $\prod_{s= \pm}\left(\hat{a}_{k, s}^{\dagger}\right)^{\nu_{k, s}}|0\rangle$ with eigenvalue $\nu_{k,+}-\nu_{k,-}$, where $|0\rangle$ is the vacuum and $\nu_{k, \pm} \in\{0,1\}$. One can think of $\hat{a}_{k, \pm}^{\dagger}$ as creating a particlehole pair of charge \pm 1 at sites $k$ and $-k$, of the form $(|01\rangle \pm|10\rangle) / \sqrt{2}$. The net charge is 0 for the states $|00\rangle$ and $|11\rangle$ [68]. It follows that the eigenstates of $\hat{C}$ are given by

$$
\left|\left\{\nu_{k, \pm}\right\}, n_{0}\right\rangle:=\left(\hat{f}_{0}^{\dagger}\right)^{n_{0}} \prod_{k=1}^{l} \prod_{s= \pm}\left(\hat{a}_{k, s}^{\dagger}\right)^{\nu_{k, s}}|0\rangle,
$$

with eigenvalue $\lambda=\nu+n_{0}-1 / 2$, where $\nu:=\sum_{k} \nu_{k,+}-\nu_{k,-}$. The integer $\nu$ measures the total charge of all Bell pairs and varies from $-l$ to $l$. Since $n_{0}$ is either 0 or $1, \lambda$ can assume $2(l+1)$ distinct values, $\{ \pm(\eta+1 / 2): \eta=0, \ldots, l\}$ with degeneracies $\left(\begin{array}{c}L \\ l-\eta\end{array}\right)$.

The eigenstates in Eq. (6) share some general features which will be inherited by the steady states. In particular, using $\left\langle\hat{f}_{k}^{\dagger} \hat{f}_{-k}\right\rangle=\left\langle\hat{C}_{k}\right\rangle / 2$ and transforming back to bosons, one finds they have an antidiagonal string order with long-range coherences, $\quad\left|\left\langle\hat{b}_{k}^{\dagger} \hat{b}_{-k}\right\rangle\right|=\left|\nu_{k,+}-\nu_{k,-}\right| / 2$, as illustrated in Fig. 1(a). The sectors labeled by $\eta=l$ are nondegenerate and maximally entangled, containing $l$ Bell pairs of the same charge, with $\left\langle\hat{b}_{k}^{\dagger} \hat{b}_{-k}\right\rangle=(-1)^{k} / 2$ [Fig. 1(b)]. It can also be shown that the reflection parity is even if $\eta$ is of the form $4 m$ or $4 m+3$ for integer $m$ and odd otherwise (see Supplemental Material [62]). The same eigenstates diagonalize $\hat{C}^{2}$ with eigenvalue $\lambda^{2}=(\eta+1 / 2)^{2}$, generating $l+1$ distinct symmetry sectors.

To find the steady states in each sector, we define $\hat{P}_{\eta}$ as the projector onto the corresponding eigenspace, $\hat{N}$ as the total particle number, and $\hat{P}_{\eta}^{\prime}:=\left(\gamma_{+} / \gamma_{-}\right)^{\hat{N}} \hat{P}_{\eta}$. Note that $\left[\hat{H}, \hat{P}_{\eta}^{\prime}\right]=0$, as $\hat{H}$ commutes with both $\hat{N}$ and $\hat{P}_{\eta}$. Furthermore, since $\hat{C}^{2}$ does not act on the center site, one

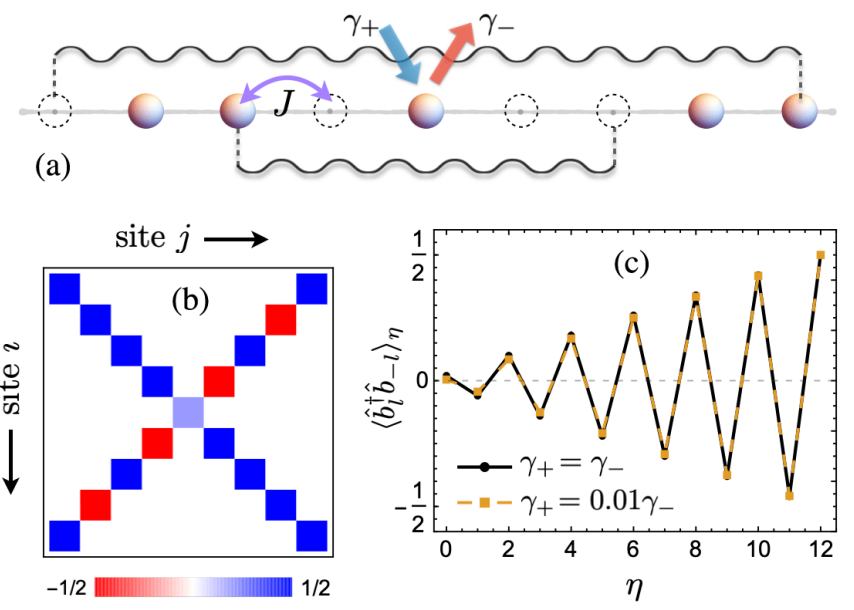

FIG. 1. (a) Schematic setup showing coherent tunneling $J$ and incoherent pump and loss $\gamma_{ \pm}$at the center. The system has a strong dynamical symmetry that stabilizes long-range entangled particle-hole pairs at reflection-symmetric sites. (b) Singleparticle density matrix $\left\langle\hat{b}_{i}^{\dagger} \hat{b}_{j}\right\rangle$ in the steady state with maximum number of Bell pairs. The center has occupation $\gamma_{+} /\left(\gamma_{+}+\gamma_{-}\right)$. (c) End-to-end coherence for different symmetry sectors $\eta$.

has the form $\hat{P}_{\eta}^{\prime}=\hat{Q}_{\eta} \otimes\left(\gamma_{+}|1\rangle\left\langle 1\left|+\gamma_{-}\right| 0\right\rangle\langle 0|\right)$, where $|0\rangle$ and $|1\rangle$ describe the center site and $\hat{Q}_{\eta}$ acts on the remaining sites. These two properties imply that $\hat{\rho}_{\eta}:=\hat{P}_{\eta}^{\prime} / \operatorname{Tr}\left(\hat{P}_{\eta}^{\prime}\right)$ is a steady state of Eq. (3) with the dissipators $\sqrt{\gamma_{+}} \hat{b}_{0}^{\dagger}$ and $\sqrt{\gamma_{-}} \hat{b}_{0}$. Numerically, we find this is the only steady state in each sector [69], up to the largest systems tractable by exact diagonalization. Within the respective eigenspace, $\hat{\rho}_{\eta}$ describes an infinite-temperature state with chemical potential $\mu=\ln \left(\gamma_{+} / \gamma_{-}\right)$. Note, however, that such a state can have high spatial entanglement, as we discuss below. For numerics, we compute $\hat{\rho}_{\eta}$ by generating all eigenstates of $\hat{C}^{2}$ by repeated applications of $\hat{a}_{k, \pm}^{\dagger}$ [Eq. (6)] and then forming $\hat{P}_{\eta}^{\prime}$. A general steady state is given by $\hat{\rho}_{\infty}=\sum_{\eta} w_{\eta} \hat{\rho}_{\eta}$ with $\sum_{\eta} w_{\eta}=1$, where $w_{\eta} \geq 0$, since $\hat{\rho}$ must be positive semidefinite. The coefficients $w_{\eta}$ can be identified as the weights $\left\langle\hat{P}_{\eta}\right\rangle$ in different symmetry sectors, which are the constants of motion. This gives a mapping from an initial state, characterized by $\left\langle\hat{P}_{\eta}\right\rangle$, to the final state [70]:

$$
\hat{\rho}_{\infty}=\sum_{\eta=0}^{l}\left\langle\hat{P}_{\eta}\right\rangle \frac{\left(\gamma_{+} / \gamma_{-}\right)^{\hat{N}} \hat{P}_{\eta}}{\operatorname{Tr}\left[\left(\gamma_{+} / \gamma_{-}\right)^{\hat{N}} \hat{P}_{\eta}\right]} .
$$

Note that $\hat{\rho}_{\infty}$ is fully determined by the weights $\left\langle\hat{P}_{\eta}\right\rangle$ and the pump-to-loss ratio, irrespective of the tunneling $J$.

Properties.-The steady states $\hat{\rho}_{\eta}$ have unique signatures in the one-particle correlations $\left\langle\hat{b}_{k}^{\dagger} \hat{b}_{-k}\right\rangle$, which can be measured experimentally [29-31] and have closed-form analytic expressions derived in Supplemental Material [62]. In particular, the end-to-end coherence grows steadily 


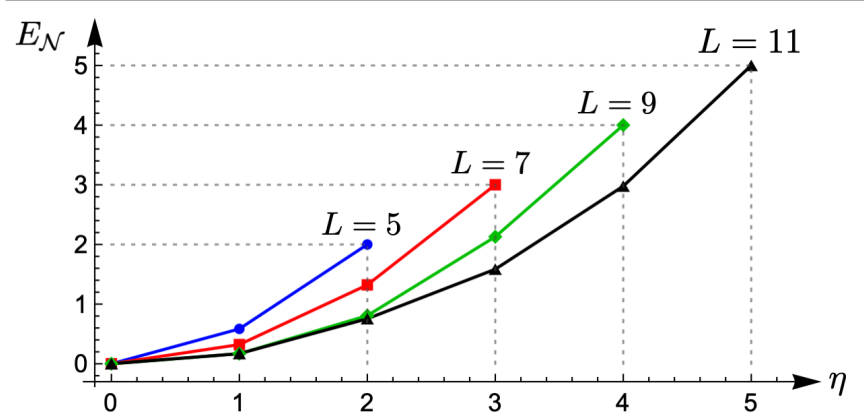

FIG. 2. Log negativity $E_{\mathcal{N}}$, measuring entanglement between left and right halves, in steady states corresponding to different symmetry sectors $\eta$ and number of sites $L$, with $\gamma_{+}=\gamma_{-}$.

with $\eta$ (in magnitude), $\left\langle\hat{b}_{l}^{\dagger} \hat{b}_{-l}\right\rangle=(-1)^{\eta}(\eta+1 / 2) / L$ for $\gamma_{+}=\gamma_{-}$, with a weak dependence on $\gamma_{+} / \gamma_{-}$, as shown in Fig. 1(c). Similar signatures appear in the density-density correlations (see Supplemental Material [62]). Note the correlations are symmetric under the exchange $\gamma_{+} \leftrightarrow \gamma_{-}$. The occupations are $\left\langle\hat{n}_{k}\right\rangle=1 / 2$ for $\gamma_{+}=\gamma_{-}$and grow monotonically with $\gamma_{+} / \gamma_{-}$, such that $\left\langle\hat{n}_{k \neq 0}\right\rangle=[1 \pm(1-$ $\eta / l)] / 2$ for $\gamma_{\mp} \rightarrow 0$.

To quantify the degree of entanglement in the (mixed) steady states, we numerically compute the log negativity $E_{\mathcal{N}}$, which gives an upper bound on the number of distillable Bell pairs between two halves of the system [71-73]. As shown in Fig. 2, it rises monotonically from $E_{\mathcal{N}}=0$ for $\eta=0$ to $E_{\mathcal{N}}=l$ for $\eta=l$. Other measures of coherence [74] give similar results (see Supplemental Material [62]).

Experimental preparation.-Preparing this system of hard-core bosons in different symmetry sectors requires a controlled generation of entanglement. As we now show, this can be done by dissipative means [75-78] if one can engineer loss of the JW fermions from the center site. For bosonic systems, such a process necessitates the application of a string operator, $\hat{f}_{0}=\left[\prod_{i<0}(-1)^{\hat{n}_{i}}\right] \hat{b}_{0}$, i.e., a boson loss accompanied by a collective phase. This can be realized efficiently in hardware with superconducting qubits coupled to ancilla cavities, as detailed in Ref. [79]. For a spin realization with cold atoms, it would be more challenging but could be implemented, in principle, with a projective measurement of the spin coupled with local Zeeman fields.

We target states in each sector that are made up solely of negatively charged Bell pairs, $\prod_{k=1}^{l}\left(\hat{a}_{k,-}^{\dagger}\right)^{\nu_{k,-}}|0\rangle$ with $\sum_{k} \nu_{k,-}=\eta$. Such states span the space of odd fermionic wave functions with occupation $N_{\text {odd }}=\eta$, i.e., a total of $\eta$ JW fermions occupying the odd modes, which are linear combinations of $\left\{\hat{a}_{k,-}^{\dagger}\right\}$ (recall that $\hat{C}=\hat{N}_{\text {even }}-\hat{N}_{\text {odd }}-1 / 2$ ). These modes are stable if one only has loss of the (now free) JW fermions at the center site. The same loss can be used to produce odd states with a given particle number, as follows. We start from a symmetric Fock state,

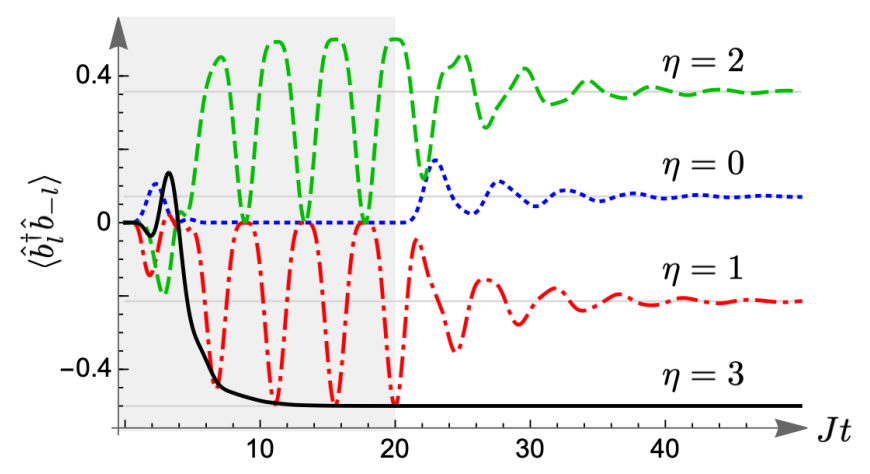

FIG. 3. End-to-end correlation during a selective preparation of different symmetry sectors $\eta$ for $L=7$. The shaded region shows the generation of Bell pairs from symmetric Fock states, $\hat{b}_{0}^{\dagger} \prod_{k=1}^{\eta} \hat{b}_{k}^{\dagger} \hat{b}_{-k}^{\dagger}|0\rangle$, driven by the loss of JW fermions at the center with rate $\gamma_{F}=3 J$. The white region shows subsequent dynamics with boson pump and loss rates $\gamma_{ \pm}=2 J$.

$\left|\left\{n_{k}\right\}\right\rangle:=\prod_{k=1}^{l}\left(\hat{b}_{k}^{\dagger} \hat{b}_{-k}^{\dagger}\right)^{n_{k}}\left(\hat{b}_{0}^{\dagger}\right)^{n_{0}}|0\rangle$. Transforming to JW fermions, one finds such a state has $N_{\text {odd }}=\sum_{k=1}^{l} n_{k}$ (see Supplemental Material [62]). Under JW fermion loss, only the even modes are depleted, so with the odd ones preserved the system will be driven to the sector $\eta=\sum_{k} n_{k}$. Thus, one can selectively prepare all different sectors simply by setting the initial occupations. In particular, a fully filled lattice evolves to the maximally entangled state, $\eta=l$.

Simulations of this preparation scheme, using exact diagonalization, are shown in Fig. 3. The oscillations describe breathing-type back-and-forth motion of the Bell pairs under the Hamiltonian. Once a given sector is prepared, one can switch from the JW fermion loss to the original boson pump and loss, converging to the steady state $\hat{\rho}_{\eta}$. Note the preparation takes a few tens of tunneling time, much faster than the on-site disorder and residual dissipation in a recent experiment [25]. We analyze these timescales further in Supplemental Material [62].

No-pump (or no-loss) limit. - If one has only boson loss at the center $\left(\gamma_{+}=0\right)$, all the odd JW fermionic modes become immune to dissipation. This is because they have no particle at the center site and are eigenmodes of $\hat{H}$. Hence, any superposition of these modes evolves unitarily under $\hat{H}$, yielding a decoherence-free subspace [32]. The same is also true for the loss of JW fermions at the center. However, the full dynamics are very different for the two cases [80]. For the boson loss, $\left\langle\hat{C}^{2}\right\rangle$ is conserved, not the occupation of odd modes, as the string in Eq. (2) couples odd and even modes. Thus, an initially filled lattice approaches the vacuum at long times, not the maximally entangled state in Fig. 3. With boson pump instead of loss, the particle and hole states are interchanged, and one again finds a decoherence-free subspace.

Robustness.-The observable $\hat{C}^{2}$ remains a generator of strong symmetry for a large class of 1D systems. First, it is 
unaffected by dephasing [43] or any Lindbladian dissipation at the center site. Second, as we show in Supplemental Material [62], $\hat{C}$ commutes with any Hamiltonian that is quadratic in the JW fermions and reflection symmetric, $[\hat{H}, \hat{R}]=0$. This includes symmetric trapping potentials, anisotropic $X Y$ spin-1/2 chains [27], and the quantum Ising model which maps onto the Kitaev chain [81]. Third, the results are unaltered for periodic boundary conditions (with an odd number of sites; see Supplemental Material [62] for more details). Furthermore, since $\left[\hat{C}, \hat{n}_{j}+\hat{n}_{-j}\right]=0$ for all $j$, any interactions or dissipation that depend only on such "pair occupations" commute with $\hat{C}$. The symmetry is, however, broken for generic dissipation away from the center and for nearest-neighbor interactions of the form $\hat{H}^{\prime}=\varepsilon \sum_{j} \hat{n}_{j} \hat{n}_{j+1}$ (see Supplemental Material [62]), as found by mapping an $X X Z$ chain to hard-core bosons. In such cases, the steady states are robust to linear order in Hamiltonian perturbations $\varepsilon[62,82]$.

Conclusions. - We have identified a paradigmatic experimental setting of a qubit array with local dissipation that exhibits a striking hidden symmetry, leading to stable longrange coherence that is both unusual and desirable. The symmetry stabilizes Bell pairs over arbitrarily long distances and is surprisingly robust. Consequently, the system has an extensive set of exactly solvable steady states characterized by an antidiagonal string order, from minimally to maximally entangled. We have shown how one can selectively prepare these states using dissipation and discern them by correlation measurements, accessible in existing photonic [25] and atomic setups. The controllable generation and preservation of long-range entanglement in an open platform would be valuable for quantum information processing and metrology $[19,73,74,83,84]$. Our findings of these special features in a simple paradigmatic model strongly motivate experimental investigations of symmetry in open systems, shedding light on the subtle relation between symmetry and conservation laws in a nonunitary setting $[15,16]$.

We thank Fabian Essler, Jon Simon, Dave Schuster, and Berislav Buča for valuable discussions. This work was supported by Engineering and Physical Sciences Research Council Grant No. EP/P009565/1 and by a Simons Investigator award.

*sd843@cam.ac.uk †nrc25@cam.ac.uk

[1] W. H. Zurek, Decoherence and the transition from quantum to classical-Revisited, Prog. Math. Phys. 48, 1 (2006).

[2] G. M. Palma, K.-A. Suominen, and A. K. Ekert, Quantum computers and dissipation, Proc. R. Soc. A 452, 567 (1996).

[3] C. H. Bennett and D. P. DiVincenzo, Quantum information and computation, Nature (London) 404, 247 (2000).
[4] M. A. Nielsen and I. L. Chuang, Quantum Computation and Quantum Information (Cambridge University Press, Cambridge, England, 2010).

[5] F. Verstraete, M. M. Wolf, and J. I. Cirac, Quantum computation and quantum-state engineering driven by dissipation, Nat. Phys. 5, 633 (2009).

[6] S. G. Schirmer and X. Wang, Stabilizing open quantum systems by Markovian reservoir engineering, Phys. Rev. A 81, 062306 (2010).

[7] S. Diehl, E. Rico, M. A. Baranov, and P. Zoller, Topology by dissipation in atomic quantum wires, Nat. Phys. 7, 971 (2011).

[8] J. F. Poyatos, J. I. Cirac, and P. Zoller, Quantum Reservoir Engineering with Laser Cooled Trapped Ions, Phys. Rev. Lett. 77, 4728 (1996).

[9] J. T. Barreiro, M. Mller, P. Schindler, D. Nigg, T. Monz, M. Chwalla, M. Hennrich, C. F. Roos, P. Zoller, and R. Blatt, An open-system quantum simulator with trapped ions, Nature (London) 470, 486 (2011).

[10] M. Müller, S. Diehl, G. Pupillo, and P. Zoller, Engineered open systems and quantum simulations with atoms and ions, Adv. At. Mol. Opt. Phys. 61, 1 (2012).

[11] A. A. Houck, H. E. Türeci, and J. Koch, On-chip quantum simulation with superconducting circuits, Nat. Phys. 8, 292 (2012).

[12] I. M. Georgescu, S. Ashhab, and F. Nori, Quantum simulation, Rev. Mod. Phys. 86, 153 (2014).

[13] L. M. Sieberer, M. Buchhold, and S. Diehl, Keldysh field theory for driven open quantum systems, Rep. Prog. Phys. 79, 096001 (2016).

[14] I. Rotter and J. P. Bird, A review of progress in the physics of open quantum systems: Theory and experiment, Rep. Prog. Phys. 78, 114001 (2015).

[15] B. Baumgartner and H. Narnhofer, Analysis of quantum semigroups with GKS-Lindblad generators: II. General, J. Phys. A 41, 395303 (2008).

[16] V. V. Albert and L. Jiang, Symmetries and conserved quantities in Lindblad master equations, Phys. Rev. A 89, 022118 (2014).

[17] B. Buča and T. Prosen, A note on symmetry reductions of the Lindblad equation: Transport in constrained open spin chains, New J. Phys. 14, 073007 (2012).

[18] V. V. Albert, B. Bradlyn, M. Fraas, and L. Jiang, Geometry and Response of Lindbladians, Phys. Rev. X 6, 041031 (2016).

[19] R. Blume-Kohout, H. K. Ng, D. Poulin, and L. Viola, Information-preserving structures: A general framework for quantum zero-error information, Phys. Rev. A 82, 062306 (2010).

[20] D. Manzano and P. I. Hurtado, Harnessing symmetry to control quantum transport, Adv. Phys. 67, 1 (2018).

[21] D. Manzano and P. I. Hurtado, Symmetry and the thermodynamics of currents in open quantum systems, Phys. Rev. B 90, 125138 (2014).

[22] J. Thingna, D. Manzano, and J. Cao, Dynamical signatures of molecular symmetries in nonequilibrium quantum transport, Sci. Rep. 6, 28027 (2016).

[23] J. Thingna, D. Manzano, and J. Cao, Magnetic field induced symmetry breaking in nonequilibrium quantum networks, New J. Phys. 22, 083026 (2020). 
[24] E. Ilievski and T. Prosen, Exact steady state manifold of a boundary driven spin-1 Lai-Sutherland chain, Nucl. Phys. B882, 485 (2014).

[25] R. Ma, B. Saxberg, C. Owens, N. Leung, Y. Lu, J. Simon, and D. I. Schuster, A dissipatively stabilized Mott insulator of photons, Nature (London) 566, 51 (2019).

[26] T. Prosen, Third quantization: A general method to solve master equations for quadratic open Fermi systems, New J. Phys. 10, 043026 (2008).

[27] T. Prosen and I. Pižorn, Quantum Phase Transition in a FarFrom-Equilibrium Steady State of an XY Spin Chain, Phys. Rev. Lett. 101, 105701 (2008).

[28] P. Kos and T. Prosen, Time-dependent correlation functions in open quadratic fermionic systems, J. Stat. Mech. (2017) 123103.

[29] S. Filipp, P. Maurer, P. J. Leek, M. Baur, R. Bianchetti, J. M. Fink, M. Göppl, L. Steffen, J. M. Gambetta, A. Blais, and A. Wallraff, Two-Qubit State Tomography Using a Joint Dispersive Readout, Phys. Rev. Lett. 102, 200402 (2009).

[30] J. G. Titchener, M. Gräfe, R. Heilmann, A. S. Solntsev, A. Szameit, and A. A. Sukhorukov, Scalable on-chip quantum state tomography, npj Quantum Inf. 4, 19 (2018).

[31] A. Bergschneider, V. M. Klinkhamer, J. H. Becher, R. Klemt, L. Palm, G. Zürn, S. Jochim, and P. M. Preiss, Experimental characterization of two-particle entanglement through position and momentum correlations, Nat. Phys. 15, 640 (2019).

[32] D. A. Lidar and K. B. Whaley, Decoherence-free subspaces and subsystems, in Irreversible Quantum Dynamics (Springer, Berlin, Heidelberg, 2003), p. 83.

[33] M. A. Cazalilla, R. Citro, T. Giamarchi, E. Orignac, and M. Rigol, One dimensional bosons: From condensed matter systems to ultracold gases, Rev. Mod. Phys. 83, 1405 (2011).

[34] G. Kordas, D. Witthaut, P. Buonsante, A. Vezzani, R. Burioni, A. I. Karanikas, and S. Wimberger, The dissipative Bose-Hubbard model, Eur. Phys. J. Special Topics 224, 2127 (2015).

[35] B. Paredes, A. Widera, V. Murg, O. Mandel, S. Fölling, I. Cirac, G. V. Shlyapnikov, T. W. Hänsch, and I. Bloch, Tonks-Girardeau gas of ultracold atoms in an optical lattice, Nature (London) 429, 277 (2004).

[36] T. Stöferle, H. Moritz, C. Schori, M. Köhl, and T. Esslinger, Transition from a Strongly Interacting 1D Superfluid to a Mott Insulator, Phys. Rev. Lett. 92, 130403 (2004).

[37] P. M. Preiss, R. Ma, M. E. Tai, A. Lukin, M. Rispoli, P. Zupancic, Y. Lahini, R. Islam, and M. Greiner, Strongly correlated quantum walks in optical lattices, Science 347, 1229 (2015).

[38] T. Matsubara and H. Matsuda, A lattice model of liquid helium, I, Prog. Theor. Phys. 16, 569 (1956).

[39] P. Jordan and E. Wigner, Über das Paulische äquivalenzverbot, Z. Phys. 47, 631 (1928).

[40] A. J. Daley, Quantum trajectories and open many-body quantum systems, Adv. Phys. 63, 77 (2014).

[41] G. Lindblad, On the generators of quantum dynamical semigroups, Commun. Math. Phys. 48, 119 (1976).

[42] V. Gorini, A. Kossakowski, and E. C. G. Sudarshan, Completely positive dynamical semigroups of $\mathrm{N}$-level systems, J. Math. Phys. (N.Y.) 17, 821 (1976).
[43] H. J. Carmichael, Statistical Methods in Quantum Optics 1 (Springer-Verlag, Berlin, 2002), Chap. 2.2.

[44] A. Rivas, A. D. K. Plato, S. F. Huelga, and M. B. Plenio, Markovian master equations: a critical study, New J. Phys. 12, 113032 (2010).

[45] A. Dhahri, A Lindblad model for a spin chain coupled to heat baths, J. Phys. A 41, 275305 (2008).

[46] M. F. Maghrebi and A. V. Gorshkov, Nonequilibrium manybody steady states via Keldysh formalism, Phys. Rev. B 93, 014307 (2016).

[47] H. Schwager, J. I. Cirac, and G. Giedke, Dissipative spin chains: Implementation with cold atoms and steady-state properties, Phys. Rev. A 87, 022110 (2013).

[48] L.-M. Duan, E. Demler, and M. D. Lukin, Controlling Spin Exchange Interactions of Ultracold Atoms in Optical Lattices, Phys. Rev. Lett. 91, 090402 (2003).

[49] M. Mamaev, I. Kimchi, R. M. Nandkishore, and A. M. Rey, Tunable spin model generation with spin-orbital coupled fermions in optical lattices, arXiv:2011.01842.

[50] A. Browaeys and T. Lahaye, Many-body physics with individually controlled Rydberg atoms, Nat. Phys. 16, 132 (2020).

[51] T. Fukuhara et al., Quantum dynamics of a mobile spin impurity, Nat. Phys. 9, 235 (2013).

[52] For $p=l$ and $q=-l$, the simplification involves using a "weak" symmetry of the Liouvillian [17], $\mathcal{L}(\hat{P} \hat{\rho} \hat{P})=$ $\hat{P}(\mathcal{L} \hat{\rho}) \hat{P} \forall \hat{\rho}$, where $\hat{P}$ is the total particle-number parity that commutes with $\hat{H}$. So the dynamics decouple into parity sectors with $\hat{P} \hat{\rho} \hat{P}= \pm \hat{\rho}$. Such weak symmetries do not imply multiple steady states but constrain their properties [53]. Other weak symmetries in our model include a paritytime reversal symmetry [54] and the particle-hole symmetry for $\gamma_{+}=\gamma_{-}$.

[53] V. Popkov and R. Livi, Manipulating energy and spin currents in non-equilibrium systems of interacting qubits, New J. Phys. 15, 023030 (2013).

[54] T. Prosen, Generic examples of PT-symmetric qubit (spin1/2) Liouvillian dynamics, Phys. Rev. A 86, 044103 (2012).

[55] H. Spohn, An algebraic condition for the approach to equilibrium of an open $N$-level system, Lett. Math. Phys. 2, 33 (1977).

[56] D. E. Evans, Irreducible quantum dynamical semigroups, Commun. Math. Phys. 54, 293 (1977).

[57] Note the steady state is unique even for the boundary-driven cases [58].

[58] T. Prosen, Comments on a boundary-driven open $X X Z$ chain: Asymmetric driving and uniqueness of steady states, Phys. Scr. 86, 058511 (2012).

[59] S. Dutta and N. R. Cooper, Out-of-equilibrium steady states of a locally driven lossy qubit array, arXiv:2007.08938.

[60] I. Pižorn, One-dimensional Bose-Hubbard model far from equilibrium, Phys. Rev. A 88, 043635 (2013).

[61] M. Cariglia, Hidden symmetries of dynamics in classical and quantum physics, Rev. Mod. Phys. 86, 1283 (2014).

[62] See Supplemental Material at http://link.aps.org/ supplemental/10.1103/PhysRevLett.125.240404 for detailed characterizations of the hidden symmetry and its eigenspaces, closed-form expressions for the steady-state correlations, coherence measures, and occupation of fermionic modes in symmetric Fock states, numerical 
estimates of preparation timescales, fidelity in the presence of dissipation at all sites, and extension to periodic boundary condition, which includes Refs. [63,64].

[63] T. Baumgratz, M. Cramer, and M. B. Plenio, Quantifying Coherence, Phys. Rev. Lett. 113, 140401 (2014).

[64] M. E. H. Ismail, Classical and Quantum Orthogonal Polynomials in One Variable (Cambridge University Press, Cambridge, England, 2005).

[65] In fact, $\hat{C}$ is the only quadratic form in the JW fermions for which this is true [62].

[66] J. E. Gough, T. S. Ratiu, and O. G. Smolyanov, Noether's theorem for dissipative quantum dynamical semi-groups, J. Math. Phys. (N.Y.) 56, 022108 (2015).

[67] K. V. Kepesidis and M. J. Hartmann, Bose-Hubbard model with localized particle losses, Phys. Rev. A 85, 063620 (2012).

[68] It is also possible to interpret $\hat{C}_{k}$ as the total $\hat{S}_{z}$ of two spin$1 / 2$ systems, where $|01\rangle \pm|10\rangle$ correspond to $|\uparrow \uparrow\rangle$ and $|\downarrow \downarrow\rangle$ and $|00\rangle$ and $|11\rangle$ correspond to $|\uparrow \uparrow\rangle \pm|\downarrow \downarrow\rangle$. Then $\hat{C}$ represents the total magnetization in the lattice.

[69] V. Popkov, S. Essink, C. Kollath, and C. Presilla, Dissipative generation of pure steady states and a gambler ruin problem, Phys. Rev. A 102, 032205 (2020).

[70] C. Sanchez Muñoz, B. Buča, J. Tindall, A. GonzálezTudela, D. Jaksch, and D. Porras, Symmetries and conservation laws in quantum trajectories: Dissipative freezing, Phys. Rev. A 100, 042113 (2019).

[71] G. Vidal and R. F. Werner, Computable measure of entanglement, Phys. Rev. A 65, 032314 (2002).

[72] M. B. Plenio and S. S. Virmani, An introduction to entanglement theory, in Quantum Information and Coherence (Springer, Cham, 2014) p. 173.

[73] R. Horodecki, P. Horodecki, M. Horodecki, and K. Horodecki, Quantum entanglement, Rev. Mod. Phys. 81, 865 (2009).
[74] A. Streltsov, G. Adesso, and M. B. Plenio, Colloquium: Quantum coherence as a resource, Rev. Mod. Phys. 89, 041003 (2017).

[75] M. B. Plenio and S. F. Huelga, Entangled Light from White Noise, Phys. Rev. Lett. 88, 197901 (2002).

[76] B. Kraus, H. P. Büchler, S. Diehl, A. Kantian, A. Micheli, and P. Zoller, Preparation of entangled states by quantum Markov processes, Phys. Rev. A 78, 042307 (2008).

[77] H. Krauter, C. A. Muschik, K. Jensen, W. Wasilewski, J. M. Petersen, J. I. Cirac, and E. S. Polzik, Entanglement Generated by Dissipation and Steady State Entanglement of Two Macroscopic Objects, Phys. Rev. Lett. 107, 080503 (2011).

[78] F. Ticozzi and L. Viola, Stabilizing entangled states with quasi-local quantum dynamical semigroups, Phil. Trans. R. Soc. A 370, 5259 (2012).

[79] G. Zhu, Y. Subaşı, J. D. Whitfield, and M. Hafezi, Hardware-efficient fermionic simulation with a cavityQED system, npj Quantum Inf. 4, 16 (2018).

[80] J. Yago Malo, E. P. L. van Nieuwenburg, M. H. Fischer, and A. J. Daley, Particle statistics and lossy dynamics of ultracold atoms in optical lattices, Phys. Rev. A 97, 053614 (2018).

[81] P. Fendley, Parafermionic edge zero modes in $Z_{n}$-invariant spin chains, J. Stat. Mech. (2012) P11020.

[82] J. Tindall, C. S. Muñoz, B. Buča, and D. Jaksch, Quantum synchronisation enabled by dynamical symmetries and dissipation, New J. Phys. 22, 013026 (2020).

[83] P. Schindler, M. Müller, D. Nigg, J. T. Barreiro, E. A. Martinez, M. Hennrich, T. Monz, S. Diehl, P. Zoller, and R. Blatt, Quantum simulation of dynamical maps with trapped ions, Nat. Phys. 9, 361 (2013).

[84] J. Huang, X. Qin, H. Zhong, Y. Ke, and C. Lee, Quantum metrology with spin cat states under dissipation, Sci. Rep. 5, 17894 (2016). 